Original research article

\title{
Protective effect of sinomenine on isoproterenol-induced cardiac hypertrophy in mice
}

\author{
$\mathrm{Le} \mathrm{Li}^{1 *}$, Pu Fang ${ }^{2}$, Jiekun Chen ${ }^{1}$, Cailing Zhang ${ }^{3}$, Houquan Tao ${ }^{4}$ \\ ${ }^{1}$ Zhejiang University of Technology, School of Pharmacy, Hangzhou, 310014, PR China \\ 2 Janssen Pharmaceuticals, Spring House, PA 19477, USA \\ ${ }^{3}$ Hangzhou Zhijiang College, Hangzhou, 310023, PR China \\ ${ }^{4}$ Lab center of Zhejiang Province People's Hospital, Hangzhou, 310014, PR China
}

\begin{abstract}
To study the effect of sinomenine (Sin) on isoproterenol (Iso, $\beta$-agonist)-induced cardiac hypertrophy ( $\mathrm{CH}$ ), we set up four mouse groups: control, Iso model, Iso+metoprolol (Met, $\beta$ blocker) $60 \mathrm{mg} / \mathrm{kg}$ and Iso+Sin $120 \mathrm{mg} / \mathrm{kg}$. CH was induced by Iso (s.c. for 28 days) in mice, and Sin or Met were orally administered by gavage for 28 days in total. Left ventricular diastolic anterior wall thickness (LVAWd), left ventricular diastolic posterior wall thickness (LVPWd), left ventricular ejection fraction (LVEF), and short axis shortening (FS) were measured by echocardiography. Malondialdehyde (MDA) and total superoxide dismutase (T-SOD) were measured by commercial kits. Lactate dehydrogenase (LDH), tumor necrosis factor-alpha (TNF- $\alpha$ ), and interleukin-1 beta (IL-1 $\beta$ ) were measured by ELISA kits. Histological changes were observed using hematoxylin-eosin (HE) and Masson staining. Protein level of nuclear transcription factorkappa B (NF- $\mathrm{kB})$ was detected by immunohistochemistry. Compared with the control group, LVAWd, Left ventricular weight index (LVWI) and myocardial fibrosis of the Iso model group significantly increased, as well as NF- $\kappa B, L D H$, MDA, TNF- $\alpha$, and IL-1 $\beta$ levels. However, the activity of T-SOD decreased. Compared with the Iso model group, LVWI of Iso model+Sin or Iso model+Met group was improved, LVAWd, LVPWd and myocardial fibrosis decreased, and NF- $\kappa B$, LDH, MDA, TNF- $\alpha$ and IL- $1 \beta$ levels decreased. T-SOD activity also increased. This study reveals that $\operatorname{Sin}$ inhibits the activation of NF- $\kappa B$, lowers the levels of TNF- $\alpha$ and IL-1 $\beta$, has anti-oxidative stress effect and inhibits myocardial inflammation in mouse heart, thereby demonstrating its efficacy in preventing Iso induced $\mathrm{CH}$.
\end{abstract}

Keywords: Cardiac hypertrophy; Echocardiography; Isoproterenol; Sinomenine; TNF- $\alpha$; IL-1 $\beta$

Highlights:

- Sin inhibited heart weight (HW), and left ventricular weight index (LVWI) of cardiac hypertrophy induced by isoprenaline in mice.

- Sin lowered serum LDH and myocardial MDA level and increased myocardial T-SOD level.

- Sin lowered serum TNF- $\alpha$, IL-1 $\beta$ level and lowered the level of NF- $\kappa$ B in myocardium.

- Sin has a protective effect on cardiac hypertrophy induced by isoprenaline in mice.

\section{Introduction}

Cardiac hypertrophy $(\mathrm{CH})$ is an adaptive response of cardiomyocytes to stress or overload volume, manifested as cardiomyocyte hypertrophy, interstitial fibrosis and abnormal electrophysiological properties. $\mathrm{CH}$ is recognized as an independent risk factor for heart failure, coronary heart disease, and sudden death (Li et al., 2014). In recent years, studies have found that the inflammatory factor plays an important role in $\mathrm{CH}$ formation (Rohini et al., 2010; Tham et al., 2015). The mechanism is complex with no effective treatments. Therefore, the search for effective prevention and treatment of $\mathrm{CH}$ has important clinical significance (Shimizu and Minamino, 2016).
Sinomenine ( $\mathrm{Sin}$ ) is monomeric alkaloid extracted from traditional Chinese medicine Coptis chinensis, which is mainly used to treat rheumatoid arthritis and arrhythmia (Liu et al., 2016). Modern pharmacology studies have demonstrated that Sin possesses wide pharmacological actions, including anti-inflammatory, anti-immune and anti-angiogenic effects (Teng et al., 2012; Wang and Li, 2011; Zhang et al., 2015). Sin might regulate immune reactions by inhibiting the activation of macrophages, peripheral blood monocytes, and microglia (Ou et al., 2011; Wang et al., 2007) and by reducing the secretion of prostaglandin E3, tumor necrosis factor-alpha (TNF- $\alpha$ ), interleukin (IL)-1 $\beta$, and interleukin (IL-6) (Xiong and Yang, 2012; Zhao et al., 2015). In addition, Sin has anti-oxidative stress effect, anti-lipid peroxidation and renoprotective features. Sin inhibits $\mathrm{H}_{2} \mathrm{O}_{2}$-induced cardiomyocyte apoptosis

\footnotetext{
* Corresponding author: Le Li, Zhejiang University of Technology, School of Pharmacy, No. 18 Chaowang Road, Hangzhou, 310014 PR China; e-mail: lile_1856@163.com http://doi.org/10.32725/jab.2021.014

Submitted: 2019-01-21 • Accepted: 2021-05-12 • Prepublished online: 2021-05-27

J Appl Biomed 19/3: 142-148 • EISSN 1214-0287 • ISSN 1214-021X

(c) 2021 The Authors. Published by University of South Bohemia in České Budějovice, Faculty of Health and Social Sciences.

This is an open access article under the CC BY-NC-ND license.
} 
(Li et al., 2008). Sin also attenuates ischemia reperfusion-induced mouse renal injuries through up-regulating Hemeoxygenase-1 (HO-1) (Zhao et al., 2013), and attenuates renal fibrosis through nuclear factor erythroid 2-related factor 2 (Nrf2)-mediated inhibition of oxidative stress and transforming growth factor $\beta$ (TGF- $\beta$ ) signaling (Qin et al., 2016). Evidence also demonstrates that Sin might be a potential agent for the treatment of osteolysis caused by Gram-negative bacteria infection or inflammation due to its inhibition of osteoclastogenesis through the reduction of Toll-like receptor 4 (TLR4)/ TNF receptor related factor 6 (TRAF6) expression and downstream signal transduction (He et al., 2016). At present, there is no research on Sin's effect on CH. So our experiments studied the effect and mechanism of $\operatorname{Sin}$ in $\beta$-agonist isoproterenol (Iso)-induced $\mathrm{CH}$ model in mice, which provides animal data for future clinical trials.

\section{Materials and methods}

\section{Experimental animals, drugs and reagents}

All aspects of the animal care and experimental protocols were in accordance with the National Institute of Health's guide for the care and use of Laboratory animals (NIH Publications No. 8023, revised 1978) and approved by the Experimental Animal Care and Use Committee of Zhejiang University of Technology. Forty-eight 8-week-old C57BL/6J male mice, weighing $19 \pm 3$ g (batch number: SCXK Zhejiang, 2015-0024), were housed in the experimental animal center of our university, where they had free access to food and drinking water. Iso in hydrochloric acid (Shanghai Hemu Pharmaceutical Co., Ltd., 161106) was used for s.c. injection. Tartrate metoprolol (Met) (batch number 170512 from AstraZeneca Pharmaceutical Co., Ltd, LDH, MDA and T-SOD were quantified by kits (Nanjing Jiancheng Research Institute). Sin was purchased from China's Xi'an grass plant technology company (XC20141120, purity

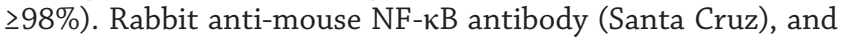
horseradish peroxidase goat anti-mouse antibody IgG (US cell signaling) were used for experiments.

\section{Instruments}

The following instruments were used for the experiments: Bio-Rad550 (US); 722N spectrophotometer (Shanghai Precision Science Instrument Co., Ltd.); KDC-2046 low temperature centrifuge (China University of Science and Technology Innovation Co., Ltd.); Qwin image analysis software (Leica, Germany).

\section{Animal models}

Forty-eight male mice were randomly divided into a control group, Iso group, Iso+Met $60 \mathrm{mg} / \mathrm{kg}$ group, and Iso+Sin $120 \mathrm{mg} / \mathrm{kg}$ group ( $n=12 /$ group). Dosage was determined by Qin et al. (2016) and our pre-experimental results. The Iso model, Iso+Met and Iso+Sin groups received s.c. Iso once a day: $40 \mathrm{mg} / \mathrm{kg}$ for the first day, $20 \mathrm{mg} / \mathrm{kg}$ for the second day, $1 \mathrm{mg} / \mathrm{kg}$ for the other 26 days (Li et al., 2003; Xu et al., 2007). Four hours after Iso administration in the morning, Met $60 \mathrm{mg} / \mathrm{kg}$ and Sin $120 \mathrm{mg} / \mathrm{kg}$ were given daily (for 28 days) to the Iso+Met and Iso+Sin groups (respectively). The control group and Iso model group were orally administered an equal volume of saline on a daily basis by gavage for 28 days.

\section{Echocardiography}

Four weeks after Iso injection, the mice were anesthetized by isoflurane inhalation ( $1.5 \%$ isoflurane in $98 \% \mathrm{O}_{2}$ ) (Shuai et al., 2019). Depilatory agents were used on the chests of the mice at constant temperature, and stabilized heart rate (400-500 beats/min). We measured left ventricular diastolic anterior wall thickness (LVAWd), left ventricular diastolic posterior wall thickness (LVPWd), left ventricular ejection fraction (LVEF), and short axis shortening (FS) using $20 \mathrm{MHz}$ high-frequency probe from Vidid7 ultrasound system (GE, USA).

\section{Determination of cardiac weight index}

Twelve hours after the last administration, all of the mice were sacrificed by stunning and cervical dislocation. Body weight (BW) was recorded. Pre-cooled saline was used to rinse thoracic cavity quickly, and heart weight (HW) was measured after dried on filter paper. Left ventricle was separated and weighed. Left ventricular weight index $(\mathrm{LVWI})=$ Left ventricular weight (LVW)/BW was calculated.

\section{Serum LDH content, TNF- $\alpha, I L-1 \beta$ levels, myocardial SOD and MDA concentrations}

The serum levels of LDH, TNF- $\alpha$ and IL- $1 \beta$ were measured by ELISA. Myocardial SOD and MDA concentrations were quantified by commercial kits (Nanjing Jiancheng Research Institute). Briefly, mouse myocardium homogenate was centrifuged at $4{ }^{\circ} \mathrm{C}, 2000 \times \mathrm{g}$ for $10 \mathrm{~min}$. The supernatant was used. All operations were strictly carried out in accordance with the manuals of the relevant kits.

\section{Myocardial histological analysis}

Middle coronal plane of the left ventricle was placed in $4 \%$ formaldehyde overnight, embedded by paraffin, sectioned, treated by alcohol gradient dehydration, subjected to HE and Masson staining, photographed under an optical microscope ( $\times 400$ ), and analyzed with Image-Pro Plus 5.0. Randomized field of Masson staining was used to calculate the blue part as myocardial fibrosis.

The expression of NF- $\kappa \mathrm{B}$ protein in myocardium was detected by immunohistochemistry. Briefly, paraffin section was dewaxed and dehydrated, immersed in $3 \%$ hydrogen peroxide for $20 \mathrm{~min}$, incubated in sheep serum for $10 \mathrm{~min}$, followed by $\mathrm{NF}-\kappa \mathrm{B}$ primary antibody incubation for $60 \mathrm{~min}$ at room temperature, goat anti-mouse IgG secondary antibody incubation for $30 \mathrm{~min}$ at room temperature, and streptavidin-oxygenase for $10 \mathrm{~min}$ at room temperature. Subsequent DAPI (4',6-diamidino-2-phenylindole) and hematoxylin staining, and film sealing were carried out, and slices were observed under a microscope. Quantitative analysis of the positive expression (brownish granules) in the cytoplasm was performed by Qwin image analysis software, and the optical density was calculated.

\section{Statistical analysis}

The results were expressed as mean \pm standard deviation (SD) and analyzed by SPSS 17.0 statistical software. Multivariate comparisons were analyzed by one-way ANOVA. The difference between the two groups was considered statistically significant if $P<0.05$.

\section{Results}

\section{Mouse model establishment}

At the beginning, the control group was well - with shiny hair and normal activity. One week after Iso administration, the mice had decreased activity and food intake. The status of the Iso+Met group and Iso+Sin group mice were better than that 
of the Iso model group. A total of 48 mice completed the experiment with 12 in each group. After 4 weeks, the mice were sacrificed. Compared with the control group, the hearts of the Iso model group had an increased weight (Table 2). Echocardiography showed that LVAWd and LVPWd in the Iso model group were significantly higher than those in the control group $(P<0.01)$, indicating that $\mathrm{CH}$ was established successfully (Table 1).
Comparison of echocardiographic results in each group As shown in Fig. 1 and Table 1, Iso model group mice had thicker LVAWd and LVPWd $(P<0.01)$, while LVEF and FS were not statistically significantly $(P>0.05)$ different compared with the control group. This suggests that overall cardiac systolic function is normal, and $\mathrm{CH}$ model was established without heart failure. Compared with the Iso model group, LVAWd and LVPWd were significantly decreased in the Iso+Met group and Iso+Sin group $(P<0.01)$.

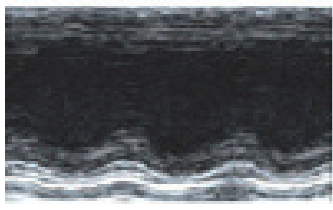

Control

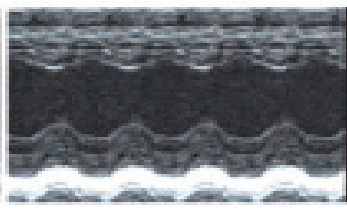

Iso model

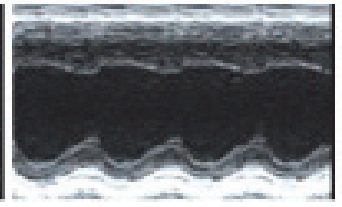

Iso+Sin $120 \mathrm{mg} / \mathrm{kg}$

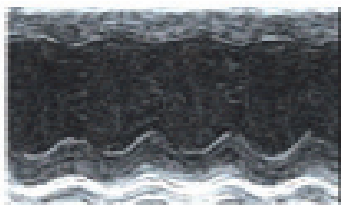

Iso+Met $60 \mathrm{mg} / \mathrm{kg}$

Fig. 1. M-mode echocardiography in each group (mean $\pm S D, n=12$ )

Table 1. Changes in echocardiographic parameters in each group (mean $\pm S D, n=12$ )

\begin{tabular}{lcccccc}
\hline Group & Dose $\mathrm{mg} / \mathrm{kg}$ & BW $(\mathrm{g})$ & LVAWd $(\mathrm{mm})$ & LVPWd $(\mathrm{mm})$ & LVEF (\%) & FS (\%) \\
\hline Control & - & $18.97 \pm 3.74$ & $1.24 \pm 0.06$ & $0.66 \pm 0.02$ & $61.9 \pm 0.02$ & $40.6 \pm 0.27$ \\
Iso model & - & $15.19 \pm 2.88^{* *}$ & $1.78 \pm 0.05^{* *}$ & $0.79 \pm 0.03$ & $60.7 \pm 0.04$ & $37.8 \pm 0.58$ \\
Iso+Sin & 120 & $17.92 \pm 2.49^{\# \#}$ & $1.58 \pm 0.05^{* * \# \#}$ & $0.73 \pm 0.06^{* * \# \#}$ & $60.9 \pm 0.03$ & $38.9 \pm 0.45$ \\
Iso+Met & 60 & $18.35 \pm 3.21^{\# \#}$ & $1.44 \pm 0.04^{* \# \# \$}$ & $0.69 \pm 0.05^{* * \# \#}$ & $61.7 \pm 0.02$ & $40.7 \pm 0.52$ \\
\hline
\end{tabular}

Note: BW: body weight; LVAWd: left ventricular diastolic anterior wall thickness; LVPWd: left ventricular diastolic posterior wall thickness;

LVEF: left ventricular ejection fraction; FS: short axis shortening. Compared with control group; ${ }^{* *} P<0.01$; compared with Iso model group;

\#\# $P<0.01$; compared with Iso+Sin group; $\$ P<0.05$.

\section{Changes of heart weight and left ventricle index in each group}

Compared with the control group, the heart weight and the left ventricle index was significantly increased in the Iso model group $(P<0.01)$. After treatment with Met $60 \mathrm{mg} / \mathrm{kg}$ or Sin $120 \mathrm{mg} / \mathrm{kg}$, LVWI was significantly lower than that in the Iso model group $(P<0.05)$, and the change in Met $60 \mathrm{mg} / \mathrm{kg}$ group was more distinct $(P<0.05)$.

Table 2. Comparison of total cardiac and left ventricular weight indexes in each group (mean $\pm S D, n=12$ )

\begin{tabular}{lcccc}
\hline Group & Dose $\mathrm{mg} / \mathrm{kg}$ & Animals & HW mg/g & LVWI mg/g \\
\hline Control & & 12 & $3.18 \pm 0.12$ & $2.25 \pm 0.16$ \\
Iso Model & - & 12 & $3.79 \pm 0.31^{*}$ & $2.97 \pm 0.21^{*}$ \\
Iso+Sin & 120 & 12 & $3.62 \pm 0.25^{\#}$ & $2.60 \pm 0.22^{\#}$ \\
Iso+Met & 60 & 12 & $3.53 \pm 0.27^{\#}$ & $2.50 \pm 0.25^{\# \$}$ \\
\hline
\end{tabular}

Note: HW: heart weight; LVWI: LVWI = LVW/BW; LVW: Left ventricle weight; BW: body weight. Compared with control group, ${ }^{*} P<0.05 ;$ compared with Iso group, ${ }^{\#} P<0.05$; compared with Iso+Sin group, $\$ P<0.05$.

\section{Serum LDH, myocardial MDA content and myocardial SOD level in each group of mice}

Serum LDH and myocardial MDA contents in the Iso model group were significantly higher than those in the control group $(P<0.01)$. The content of LDH and MDA in the Iso+Met and Iso+Sin group were significantly lower than those in the Iso model group, and the myocardial SOD level was significantly increased $(P<0.05)$. The effect of Met $60 \mathrm{mg} / \mathrm{kg}$ was stronger (Fig. 2).

\section{TNF- $\alpha$ and IL-1 $\beta$ levels in each group}

Compared with the control group, the levels of TNF- $\alpha$ and IL-1 $\beta$ in Iso model group were significantly higher $(P<0.05)$. Compared with the Iso model group, TNF- $\alpha$ and IL-1 $\beta$ levels were significantly decreased in the Iso+Met group and Iso+Sin group $(P<0.05)$. Compared with the Iso+Sin group, the change in Iso+Met $60 \mathrm{mg} / \mathrm{kg}$ group was more distinct $(P<0.05)$ (Table 3). 

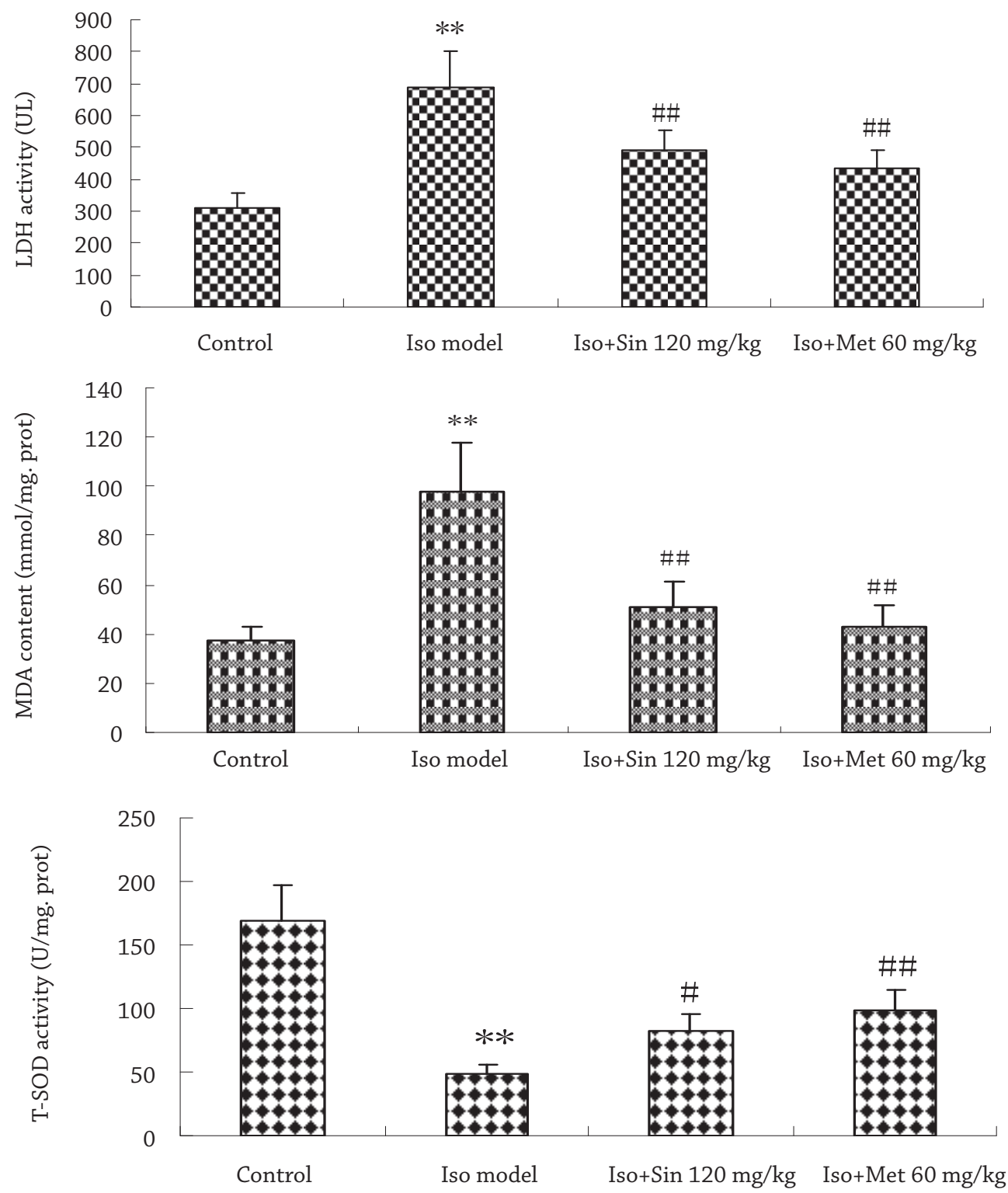

Fig. 2. Changes of serum LDH content, myocardial MDA content and SOD level in each group (mean $\pm S D, n=12)$. Compared with the control group, ${ }^{* *} P<0.01$; compared with Iso model group; $\#<<0.05$; \#\# $P<0.01$.

Table 3. The levels of TNF- $\alpha$ and IL-1 $\beta$ in the serum of the four groups (mean $\pm S D, n=12$ )

\begin{tabular}{lccc}
\hline Group & $\begin{array}{c}\text { Dose } \\
\mathrm{mg} / \mathrm{kg}\end{array}$ & $\begin{array}{c}\text { TNF- } \alpha \\
\mu \mathrm{g} / \mathrm{l}\end{array}$ & $\begin{array}{c}\mathrm{IL}-1 \beta \\
\mu \mathrm{g} / \mathrm{l}\end{array}$ \\
\hline Control & - & $1.68 \pm 0.38$ & $0.20 \pm 0.03$ \\
Iso Model & - & $3.89 \pm 0.56^{*}$ & $0.46 \pm 0.6^{*}$ \\
Iso+Sin & 120 & $2.34 \pm 0.43^{* \#}$ & $0.27 \pm 0.04^{* \#}$ \\
Iso+Met & 60 & $1.96 \pm 0.27^{* \# \$}$ & $0.25 \pm 0.04^{* \# \$}$ \\
\hline
\end{tabular}

Note: TNF- $\alpha$ : tumor necrosis factor-alpha; IL-1 $\beta$, interleukin-1 beta. Compared with the control group; ${ }^{*} P<0.05$; compared with Iso model group; ${ }^{\#} P<0.05$; compared with Iso+Sin model group; ${ }^{\$} P<0.05$.

\section{Myocardial histopathology observation}

Light microscopy showed that the myocardial cells were arranged neatly, and the cytoplasm was evenly stained in control group. The Iso group had increased size of myocardial cells, increased staining of the nucleus, increased fibrosis indicated by intercellular blue staining, and increased inflammatory cell infiltration. After Met $60 \mathrm{mg} / \mathrm{kg}$ or Sin $120 \mathrm{mg} / \mathrm{kg}$ treatment, cardiomyocytes are arranged neatly, and the degree of myocardial hypertrophy is reduced, inflammatory cell infiltration decreased and interstitial fibrosis is significantly reduced (Figs 3, 4).

\section{NF- $\boldsymbol{\kappa} B$ protein expression in myocardium}

Fig. 5 and Fig. 6 show that total NF-кB protein expression level was significantly up-regulated in the Iso group compared with the control group $(P<0.01)$. The expression of NF- $\kappa \mathrm{B}$ protein was significantly down-regulated in the Iso+Sin group or the Iso+Met group $(P<0.01)$. 


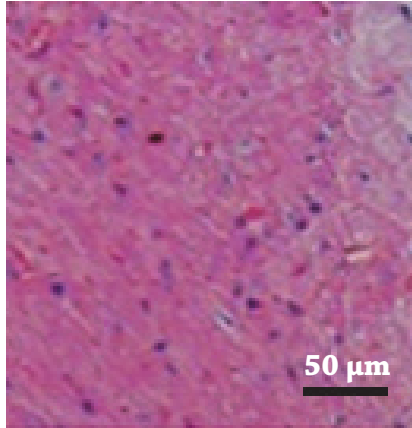

Control

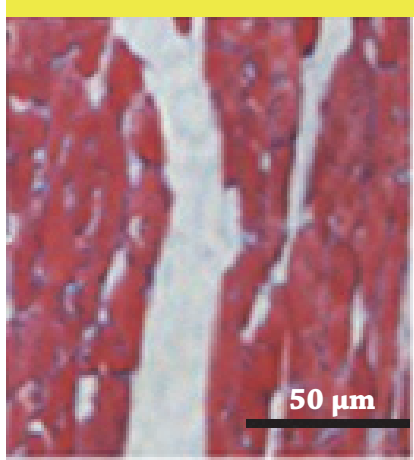

Control

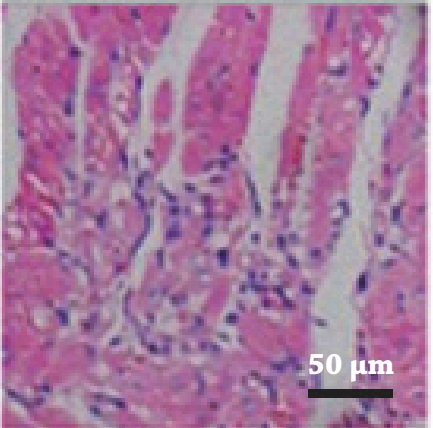

Iso model

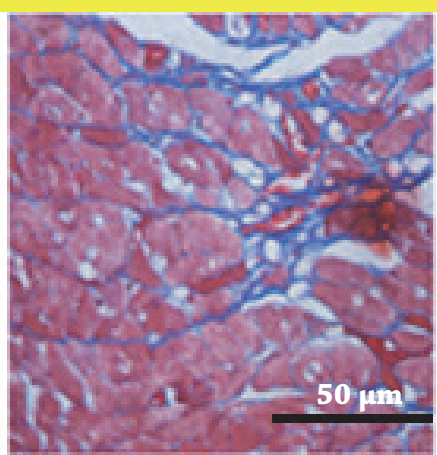

Iso model

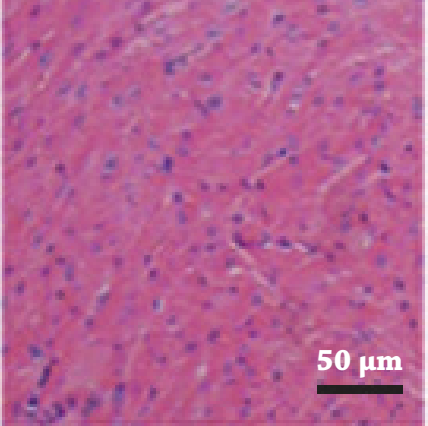

Iso+Sin 120 mg/kg

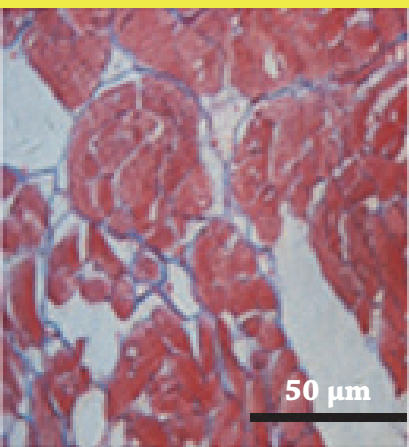

Iso+Sin $120 \mathrm{mg} / \mathrm{kg}$

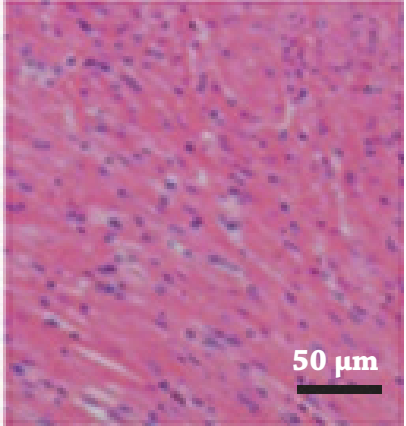

Iso+Met $60 \mathrm{mg} / \mathrm{kg}$

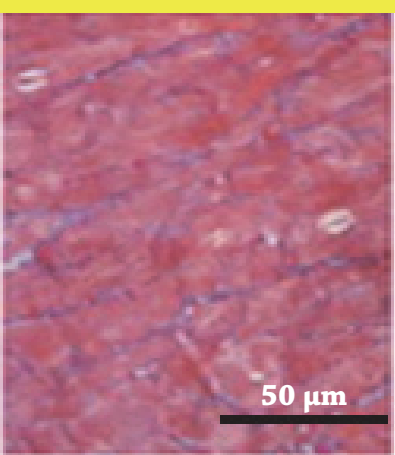

Iso+Met $60 \mathrm{mg} / \mathrm{kg}$

Fig. 3. Histopathological changes of myocardium in mice (Top: HE staining $\times 200$; Bottom: Masson staining $\times 400)$

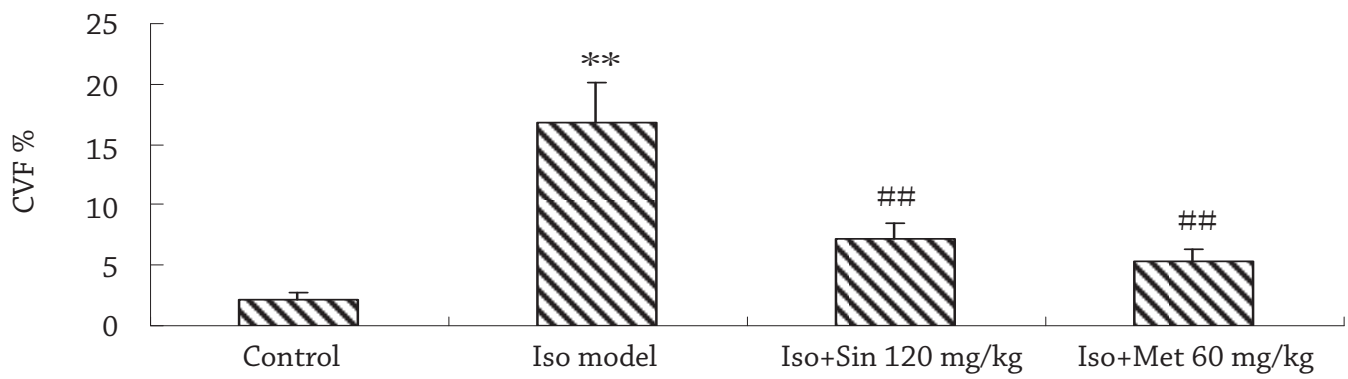

Fig. 4. Changes of myocardial tissue fibrosis in each group (mean $\pm S D, n=12$ ). Compared with the control group; ** $P<0.01$; compared with the Iso model group; \# $P<0.05$; \#\# $P<0.01$.

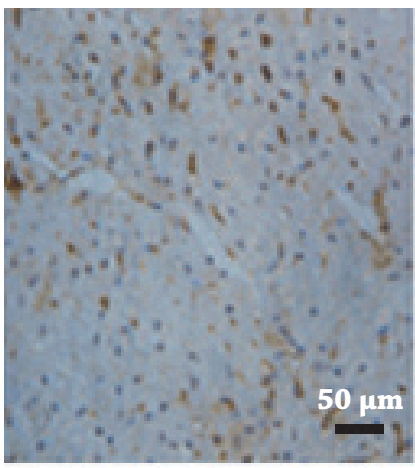

Control

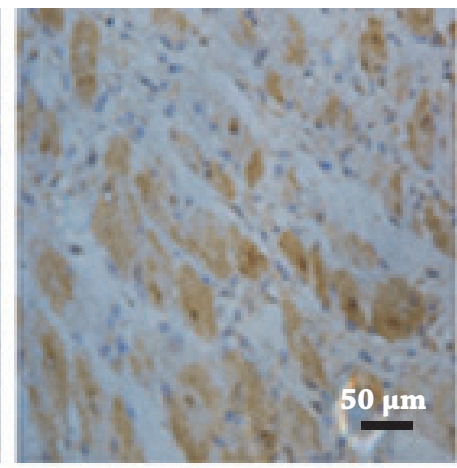

Iso model

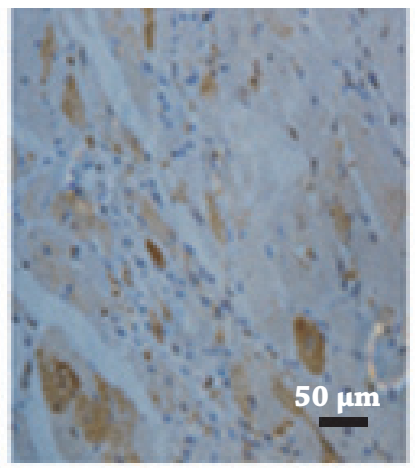

Iso+Sin $120 \mathrm{mg} / \mathrm{kg}$

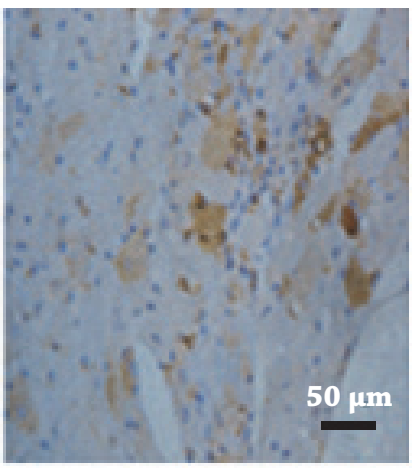

Iso+Met $60 \mathrm{mg} / \mathrm{kg}$

Fig. 5. The expression of NF-kB protein in myocardium of mice in each group (mean $\pm S D, n=12, \times 100$ ) 


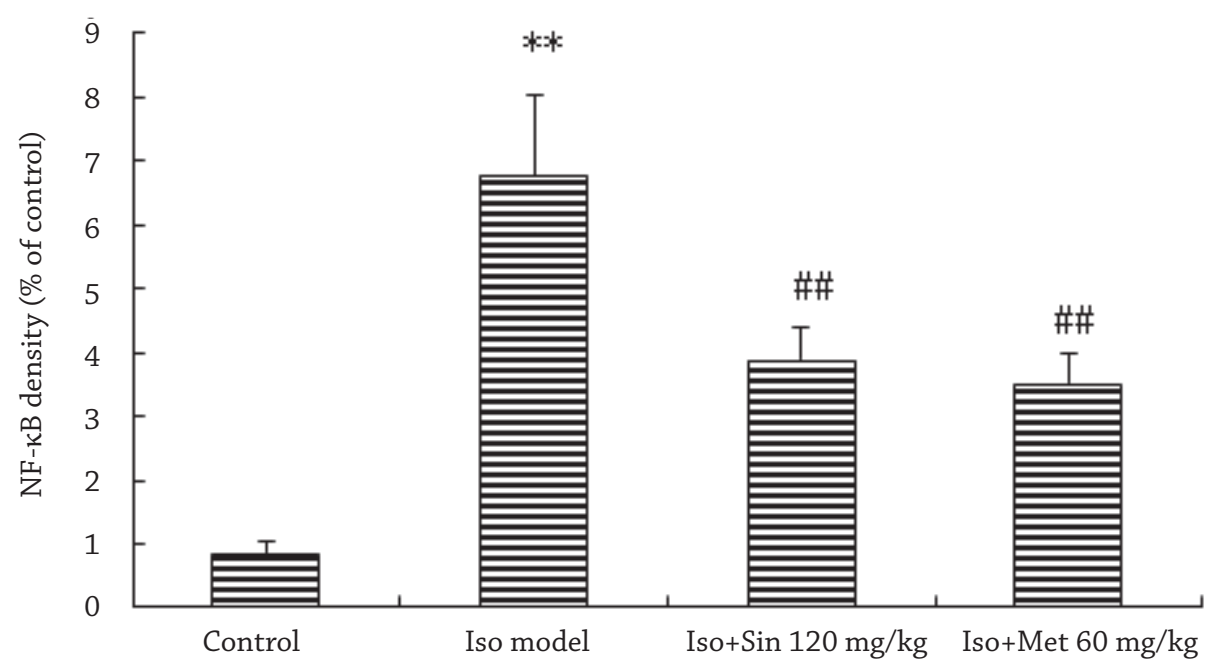

Fig. 6. Changes of NF- $\kappa B$ protein expression in myocardium of mice (mean $\pm S D, n=12$ ). Compared with the control group; ${ }^{* *} P<0.01$; compared with the Iso model group; ${ }^{\#} P<0.01$.

\section{Discussion}

$\mathrm{CH}$ is a common pathological process in many cardiovascular diseases, characterized by cardiomyocyte hypertrophy and interstitial component changes (myocardial remodeling) (Li et al., 2014; Tham et al., 2015). A previous study found that hypertrophic myocardium is a serious inflammatory response, which increases myocardial damage, myocardial remodeling, and ultimately leads to heart failure (Zheng et al., 2004). The aim of the present study was to investigate the expression of inflammatory cytokines in myocardium induced by Iso, and to explore the protective effect and mechanisms of $\operatorname{Sin}$ on $\mathrm{CH}$.

Iso is a $\beta$ adrenoreceptor agonist, which speeds up heart rate, accelerates neural conduction, increases myocardial oxygen consumption, and increases cyclic adenosine monophosphate synthesis and glycogen synthesis, thereby promoting the myocardium total protein and non-contractile protein synthesis, enhancing myocardial contractility, and resulting in cardiomyocyte hypertrophy and collagen deposition (Yang et al., 2013). These changes are the specific phenotype of $\mathrm{CH}$ (Heidecker and Hare, 2008). Meanwhile, circulation and cardiac catecholamines increase, causing lipid metabolism abnormalities and oxygen free radical increases (Li et al., 2014; Shimizu and Minamino, 2016). In this study, we established an Iso-induced $\mathrm{CH}$ mouse model, which is confirmed by ultrasound observation of left ventricular wall thickening and normal left ventricular ejection fraction in mice. LVWI increases, combined with HE staining, demonstrated myocardial hypertrophy. Masson staining showed myocardial fiber thickening and interstitial fibrosis increase, suggesting that Iso induced $\mathrm{CH}$ model successfully. Our results also showed that Sin or Met reduced the degree of $\mathrm{CH}$ (reduced myocardial hypertrophy and interstitial fibrosis) in Iso-administrated mice and decreased LVWI. Met was used as a positive control for the treatment of $\mathrm{CH}$. The effect of Met was consistent with the existing literature reports (Hanada et al., 2008).

MDA is a free radical induced by lipid peroxidation. SOD is a superoxide dismutase, which is an important enzyme for defending superoxide ions both intracellularly and extracellularly. Increased MDA content and decreased SOD activity can lead to oxidative stress (Rizzi et al., 2014). Our results indicated that Sin lowered MDA content, and increased myocardial SOD activity, showing that Sin is a good antioxidant and myocardial protectant.

A large number of inflammatory mediators, such as NF$\kappa B$, TNF- $\alpha$, IL- $1 \beta$ etc, and their signaling pathways are activated locally in $\mathrm{CH}$ (Lu et al., 2016; Young et al., 2008). NF- $\kappa B$ stimulates the expression of cytokines such as TNF- $\alpha$ and IL-1 $\beta$ (Shimizu and Minamino, 2016). Thus, NF- $\kappa B$ activation can promote cardiac formation $\mathrm{CH}$ through its inflammation induction property, and inhibition of NF- $\mathrm{BB}$ can effectively inhibit $\mathrm{CH}$ and inflammation in myocardial injury (Gullestad et al., 2012). Similar to TNF- $\alpha$, IL-1 $\beta$ expression was positively correlated with the degree of left ventricular hypertrophy (Maulik and Kumar, 2012; Shimizu and Minamino, 2016). In recent years, clinical studies have shown that Sin has a definite safe and curative effect on the mesangial proliferative glomerulonephritis, IgA nephropathy and diabetic nephropathy. The regulation of immunity and anti-inflammation is considered to be the main pharmacological basis for its role in the prevention and treatment of nephropathy (Teng et al., 2019). This study found that $\operatorname{Sin}$ (or Met) reduces the levels of inflammatory cytokines such as TNF- $\alpha$ and IL-1 $\beta$ by inhibiting the activation of NF- $\kappa \mathrm{B}$, reduces the inflammatory response in myocardium, inhibits ventricular wall thickening, reduces myocardial hypertrophy and collagen production, and improves cardiac cavity expansion, and ventricular hypertrophy. Combined with our experimental data, it is reasonable to speculate that $\mathrm{Sin}$ may alleviate the $\mathrm{CH}$ through antioxidant and anti-inflammatory effects. The role of Met was consistent with the existing literature reports (Hanada et al., 2008) and the role of Met was stronger than that of Sin. At present, Sin is clinically used to treat rheumatic diseases (Liu et al., 2016). Based on the experimental results, we believe that Sin may become a clinical drug candidate for the treatment of $\mathrm{CH}$ in the future. In the follow-up, we shall further study Sin's mechanism and action on $\mathrm{CH}$, hoping to provide strong animal experimental evidence for future clinical applications. 


\section{Conclusions}

In summary, Sin inhibits the activation of $N F-\kappa B$ and down-regulates the levels of inflammatory cytokines such as TNF- $\alpha$ and IL-1 $\beta$, reduces the inflammatory reaction in myocardial tissue, inhibits ventricular wall thickening, and reduces myocardial hypertrophy and collagen production. Sin may have better clinical therapeutic significance for cardiovascular diseases such as hypertension, valvular heart disease, hyperthyroidism, anemia and other diseases, which are characterized by the $\mathrm{CH}$ as a typical pathology for them.

\section{Acknowledgements}

This work was supported by project grants from the Natural Science Foundation of Zhejiang province in China (LY13H310005) and in part by project grants from the Key Natural Science Foundation of Zhejiang University of Technology in China (X1168117).

\section{Conflict of interests}

All authors declare that they have no conflict of interests.

\section{References}

Gullestad L, Ueland T, Vinge LE, Finsen A, Yndestad A, Aukrust P (2012). Inflammatory cytokines in heart failure: mediators and markers. Cardiology 122(1): 23-35. DOI: $10.1159 / 000338166$.

Hanada K, Asari K, Saito M, Kawana J-I, Mita M, Ogata H (2008). Comparison of pharmacodynamics between carvedilol and metoprolol in rats with isoproterenol-induced cardiac hypertrophy: effects of carvedilol enantiomers. Eur J Pharmacol 589(1-3): 194-200. DOI: 10.1016/j.ejphar.2008.04.055.

He L, Duan H, Li X, Wang S, Zhang Y, Lei L, et al. (2016). Sinomenine down-regulates TLR4/TRd osteolysis. Eur J Pharmacol 779: 66-79. DOI: 10.1016/j.ejphar.2016.03.014.

Heidecker B, Hare JM (2008). Cardiovascular genetic medicine: genomic assessment of prognosis and diagnosis in patients with cardiomyopathy and heart failure. J Cardiovasc Transl Res 1(3): 225-231. DOI: 10.1007/s12265-008-9044-y.

Li L, Gao X-L, Ding B-X (2008). Inhibitory effect of sinomenine on H2O2-induced apoptosis in neonatal rat cardiomyocytes. Zhongguo Zhong Yao Za Zhi 33(8): 939-941.

Li L, Shang H, Zhang C, Tao H, Cheng J (2014). Experimental study of fasudil on the myocardial hypertrophy of the transverse aortic constriction in rats. Lat Am J Pharm 33(10): 1618-1623.

Li M, Zhang J, Chen Y, Wang YQ (2003). Danshen inhibiting isoproterenol induced cardiac hypertrophy and fibrosis in mice and its mechanisms. J China Pharm Univ (Chin) 34: 565-568.

Liu W, Qian X, Ji W, Lu Y, Wei G, Wang Y (2016). Effects and safety of Sinomenine in treatment of rheumatoid arthritis contrast to methotrexate: a systematic review and Meta-analysis. Tradit Chin Med 36(5): 564-577. DOI: 10.1016/s0254-6272(16)30075-9.

Lu J, Liu F, Chen F, Jin Y, Chen H, Liu D, Cui W (2016). Amlodipine and atorvastatin improve ventricular hypertrophy and diastolic function via inhibiting TNF- $\alpha, \mathrm{IL}-1 \beta$ and $N F-\kappa B$ inflammatory cytokine networks in elderly spontaneously hypertensive rats. Biomed Pharmacother 83: 330-339. DOI: 10.1016/j. biopha.2016.06.034.

Maulik SK, Kumar S (2012). Oxidative stress and cardiac hypertrophy: a review. Toxicol Mech Methods 22(5): 359-366. DOI: 10.3109/15376516.2012.666650

Ou Y, Li W, Li X, Lin Z, Li M (2011). Sinomenine reduces invasion and migration ability in fibroblast-like synoviocytes cells co-cultured with activated human monocytic THP-1 cells by inhibiting the expression of MMP-2, MMP-9, CD147. Rheumatol Int 31(11): 1479-1485. DOI: 10.1007/s00296-010-1506-2.

Qin T, Yin S, Yang J, Zhang Q, Liu Y, Huang F, Cao W (2016). Sinomenine attenuates renalfibrosis through Nrf2-mediated inhibition of oxidative stress and TGF $\beta$ signaling. Toxicol Appl Pharmacol 304: 1-8. DOI: 10.1016/j.taap.2016.05.009.

Rizzi E, Guimaraes DA, Ceron CS, Prado CM, Pinheiro LC, Martins-Oliveira A, et al. (2014). $\beta 1$-Adrenergic blockers exert antioxidant effects, reduce matrix metalloproteinase activity, and improve renovascular hypertension-induced cardiac hypertrophy. Free Radic Biol Med 73: 308-317. DOI: 10.1016/j. freeradbiomed.2014.05.024.

Rohini A, Agrawal N, Koyani CN, Singh R (2010). Molecular targets and regulators of cardiac hypertrophy. Pharmacol Res 61(4): 269-280. DOI: 10.1016/j.phrs.2009.11.012.

Shimizu JT, Minamino J (2016). Physiological and pathological cardiac hypertrophy. J Mol Cell Cardiol 97: 245-262. DOI: 10.1016/j.yjmcc.2016.06.001.

Shuai W, Kong B, Fu H, Shen C, Jiang X, Huang H (2019). MD1 deficiency promotes inflammatory atrial remodelling induced by high-fat diets. Can J Cardiol 35(2): 208-216. DOI: 10.1016/j. cjca.2018.11.020.

Teng J, Zhao LJ, Zhou ZH, Zhou H, Huang SL, Chou P, et al. (2019). Research Progress in the Treatment of Kidney Diseases with Sinomenine Hydrochloride. Guid J Trad Chin Med Pharm 25(16): 120-124.

Teng P, Liu H-L, Zhang L, Feng L-L, Huai Y, Deng Z-S, et al. (2012). Synthesis and biological evaluation of novel sinomenine derivatives as anti-inflammatory agents. Eur J Med Chem 50: 63-74. DOI: 10.1016/j.ejmech.2012.01.036.

Tham YK, Bernardo BC, Ooi JYY, Weeks KL, McMullen JR (2015). Pathophysiology of cardiac hypertrophy and heart failure: signaling pathways and novel therapeutic targets. Arch Toxicol 89(9): 1401-1438. DOI: 10.1007/s00204-015-1477-x.

Wang AL, Li Z, Yuan M, Yu ACH, Zhu X, Tso MOM (2007). Sinomenine inhibits activation of rat retinal microglia induced by advanced glycation end products. Int Immunopharmacol 7(12): 1552-1558. DOI: 10.1016/j.intimp.2007.07.030.

Wang Q, Li X-K (2011). Immunosuppressive and anti-inflammatory activities of sinomenine. Int Immunopharmacol 11(3): 373-376. DOI: 10.1016/j.intimp.2010.11.018.

Wu L, Qiao H, Li Y, Li D (2007). Cardioprotective effects of the combined use of puerarin and Danshensu on acute ischemic myocardial injury in rats. Phytother Res 21(8): 751-756. DOI: $10.1002 /$ ptr.2157.

Xiong L, Yang L (2012). Effects of alkaloid sinomenine on levels of IFN- $\gamma$, IL-1 $\beta$, TNF- $\alpha$ and IL- 6 in a rat renal allograft model Immunotherapy 4(8): 785-791. DOI: 10.2217/imt.12.80.

Xu X-L, Ji H, Gu SY, Huang Q-J, Chen Y-P (2007). Protective effects of astragaloside on isoproterenol induced cardiac hypertrophy in mice. J China Pharm Univ (Chin) 38(5): 451-455.

Yang J, Wang H-X, ZhangY-J, Yang Y-H, Lu M-L Zhang J, et al. (2013). Astragaloside IV attenuates inflammatory cytokines by inhibiting TLR4/NF- $\mathrm{B}$ signaling pathway in isoproterenolinduced myocardial hypertrophy. J Ethnopharmacol 150(3): 1062-1070.

Young D, Popovic ZB, Jones WK, Gupta S (2008). Blockade of NF-kappaB using IkappaB alpha dominant-negative mice ameliorates cardiac hypertrophy in myotrophin-overexpressed transgenic mice. J Mol Biol 381(3): 559-568. DOI: 10.1016/j. jmb.2008.05.076.

Zhang HM, Ren Y, Tang X, Wang K, Liu Y, Zhang L, et al. (2015). Vascular normalization induced by sinomenine hydrochloride results in suppressed mammary tumor growth and metastasis. Sci Rep 5: 8888. DOI: $10.1038 /$ srep08888.

Zhao Z, Guan R, Song S, Zhang M, Liu F, Guo M, et al. (2013). Sinomenine protects mice against ischemia reperfusion induced renal injury by attenuating inflammatory response and tubular cell apoptosis. Int J Clin Exp Pathol 6(9): 1702-1712.

Zhao Z, Xiao J, Wang J, Dong W, Peng Z, An D (2015). Antiinflammatory effects of novel sinomenine derivatives. Int Immunopharmacol 29(2): 354-360. DOI: 10.1016/j. intimp.2015.10.030.

Zheng M, Han Q-D, Xiao R-P (2004). Distinct $\beta$-adrenergic receptor sub-type signaling in the heart and their payhophysiological relevance. Sheng Li Xue Bao 56(1): 1-15. 